\title{
MULTI-SENSOR DATA FUSION USING GEOMETRIC TRANSFORMATIONS FOR GAS TRANSMISSION PIPELINE INSPECTION
}

\author{
Joseph A. Oagaro and Shreekanth Mandayam, Senior Member, IEEE \\ Department of Electrical \& Computer Engineering \\ Rowan University \\ 201 Mullica Hill Road, Glassboro, NJ 08028
}

\begin{abstract}
This paper presents a technique that can be used to fuse data from multiple sensors that are employed in nondestructive evaluation (NDE) applications, specifically for the in-line inspection of gas transmission pipelines. A radial basis function artificial neural network is used to perform geometric transformations on data obtained from multiple sources. The technique allows the user to define the redundant and complementary information present in the data sets. The efficacy of the algorithm is demonstrated using experimental images obtained from the NDE of a test specimen suite using magnetic flux leakage (MFL), ultrasonic (UT) and thermal imaging methods. The results presented in this paper indicate that neural network based geometric transformation algorithms show considerable promise in multi-sensor data fusion applications.
\end{abstract}

Keywords - Image processing, pattern recognition, industrial monitoring, inverse problems

\section{INTRODUCTION}

Nondestructive evaluation (NDE) plays a vital role in the operation and maintenance of large infrastructure such as gas transmission pipelines, nuclear power plants, aircraft, bridges and highways, etc. As this infrastructure continues to age it is essential that the inspection techniques reliably and accurately predict the integrity of these systems [1]. No single NDE method is capable of inspecting everything and extracting all required information - a combination of methods must be used and the resulting data fused to extract relevant information. Moreover, newer systems that are developed are often made of composite materials that include metals and dielectrics. One interrogation modality cannot be used to inspect such components for reliability - multiple tests are always needed. The accurate interpretation of inspection data obtained from multiple methods requires the development of multi-sensor data fusion algorithms. The most common interpretation result expected from an NDE procedure is information about the location, size and shape of structural defects in the component.

A variety of techniques have been explored for fusing NDE data from multiple sources. NDE image data from homogeneous and heterogeneous sources have been fused both in spatial and spectral domains. An example of homogeneous data fusion is a technique developed to combine multi-frequency eddy current images of the same test specimen [2]. The authors used discrete cosine transforms to fuse the two images in the transform domain. An example of heterogeneous data fusion is a technique to combine an eddy current image and an infrared thermal image of the same test specimen [3]. The authors used a total of six pixel-level data fusion techniques: maximum amplitude, integration, averaging, weighted averaging, Bayesian analysis and DempsterShafer theory. Ultrasonic and eddy-current NDE images have been combined using Bayesian analysis [4]; linear minimum mean square error (LMMSE) filter [5] and image morphological techniques [6].

The efficacy of the various data fusion algorithms have typically been demonstrated by showing that the fused image contains features that cannot be discerned in the original NDE images that are input to the algorithm. There have not been sufficient attempts to define quantitative measures for this purpose. In this paper, we attempt to address the measurement of the effectiveness of a data fusion technique by explicitly defining the information expected as a result of the fusion process.

\section{APPROACH}

When NDE images that are obtained from different inspection methods are fused, the fused image can be assumed to contain two main types of information that are 
related to the characteristics of the test object: redundant and complementary information. Redundant information is the information related to the defect that is common among different inspection methods and can be used to increase the reliability of the defect characterization result. Complementary information is the defect related information that is unique to each inspection method and can be used to improve the accuracy of defect characterization. Figure 1 pictorially depicts the redundant and complementary information in the data fusion process.

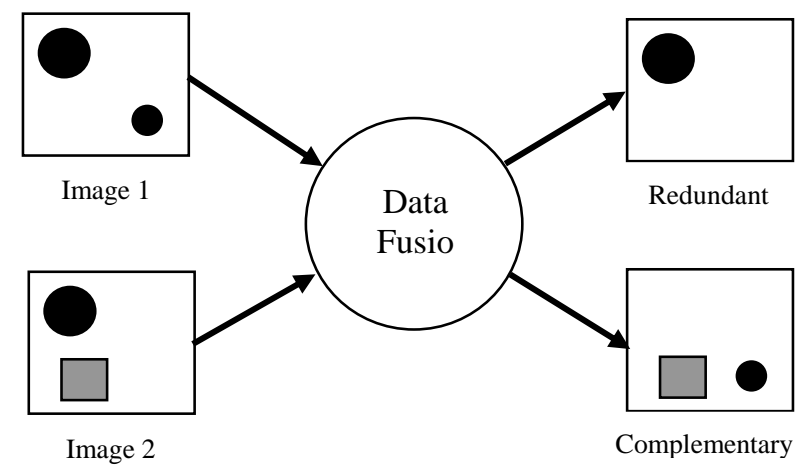

Figure 1: Pictorial depiction of redundant and complementary information in data fusion.

The data fusion technique described in this paper employs geometric transformations for extracting redundant and complementary information between pairs of NDE inspection images. Geometric transformations are typically used in image processing for correcting the effects of spatial distortion in images [7]. In this paper, radial basis function artificial neural networks are employed for performing geometric transformations [8]. The neural networks serve two purposes - they are universal approximators; and the ability to train them allows for the explicit definition of redundant and complementary information among multiple data sets.

Let $x_{1}\left(r_{1}, c_{1}\right)$ and $x_{2}\left(r_{1}, c_{2}\right)$ be two different NDE images that are the results of the inspection of the same object using two different inspection modalities. The variable $r$ represents the redundant information features and is the same for both images. Likewise, the variables $c_{1}$ and $c_{2}$ represent the complementary information features for each image. A function that extracts the redundant defect related information, $h_{1}(r)$, between $x_{1}\left(r_{1}, c_{1}\right)$ and $x_{2}\left(r_{1}, c_{2}\right)$ can be defined as:

$$
f\left\{x_{1}\left(r, c_{1}\right), x_{2}\left(r, c_{2}\right)\right\}=h_{1}(r)
$$

If two arbitrary functions $g_{1}$ and $g_{2}$ are defined, $h_{1}(r)$ can be obtained using the following equation:

$$
h_{1}(r) \diamond g_{1}\left(x_{1}\right)=g_{2}\left(x_{2}\right)
$$

where $\diamond$ represents a homomorphic operator. For this case, the homomorphic operator was chosen to be the addition operator, + . Therefore, Equation (2) becomes:

$$
h_{1}(r)+g_{1}\left(x_{1}\right)=g_{2}\left(x_{2}\right)
$$

In order to use the technique defined by Equation (3), the three arbitrary functions $h_{1}(r), g_{1}$ and $g_{2}$ must be determined. The function $h_{1}(r)$ is chosen depending on the needs of the user. The function $g_{2}$ is defined as a conditioning function and is an application-dependant function that may be used to condition the data to better suit the application. An example of this is if the data values within $x_{2}$ have spread over a wide range, $g_{2}$ may be chosen to be a logarithmic function. If $h$ and $g_{2}$ are specified, a universal approximation technique may be used to determine the function that maps $g_{1}$ to the rest of the expression in Equation (4).

$$
g_{1}\left(x_{1}\right)=g_{2}\left(x_{2}\right)-h_{1}(r)
$$

Ideally, a radial basis function will produce the best function approximation of $g_{1}$ given the proper training data. If the conditioning function $g_{2}$ is assumed to be an identity function, Equation (4) can be simplified to:

$$
g_{1}\left(x_{1}\right)=x_{2}-h_{1}(r)
$$

where $x_{1}$ is the training input of the RBF and the expression $x_{2}-h_{1}(r)$ is the training output.

After the RBF neural network has been trained with an appropriate training data set, the network is ready to receive the testing data set. The testing procedure is described as:

$$
h_{1}(r)=x_{2}-g_{1}\left(x_{1}\right)
$$

The RBF neural network is fed the testing $x_{1}$ data as in the training sequence. However, the output of the network $x_{2}-h_{1}(r)$ is inverted and $x_{2}$ is subtracted from the inverted output. Therefore, the redundant data is effectively extracted resulting in $h_{1}(r)$ as the final output.

The complementary information extraction technique follows a mathematical process that is almost identical to the redundant data extraction technique. Equation (1) becomes:

$$
f\left\{x_{1}\left(r, c_{1}\right), x_{2}\left(r, c_{2}\right)\right\}=h_{2}\left(c_{1}, c_{2}\right)
$$

where $h_{1}(r)$ has been replaced with $h_{2}\left(c_{1}, c_{2}\right)$. Also, the RBF neural network whose output is denoted as $g_{1}$ is different for the complementary data extraction technique since the network has been trained with complementary, not redundant, data. 


\section{IMPLEMENTATION RESULTS}

A suite of test specimens was fabricated to mimic defects arising from pitting corrosion that occurs in underground gas transmission pipelines. The specimens were subjected to three kinds of NDE: ultrasonic testing (UT), magnetic flux leakage (MFL) and thermal imaging. Defect signature images resulting from inspecting these specimens are combined in pairs using the data fusion algorithm described in this paper. The definitions of redundant and complementary information can be made by comparing the NDE signature for each of the inspection methods with the actual defect characteristics, which are known, for the specimen suite. Figure 2 illustrates this definition process. Complementary information in two NDE images are defined as those distinct pixels in each of the NDE signatures that are present in the defect region, but are not shared between them. Redundant information in two NDE images are defined as those common pixels that are present in both NDE signatures and are also present in the defect region.

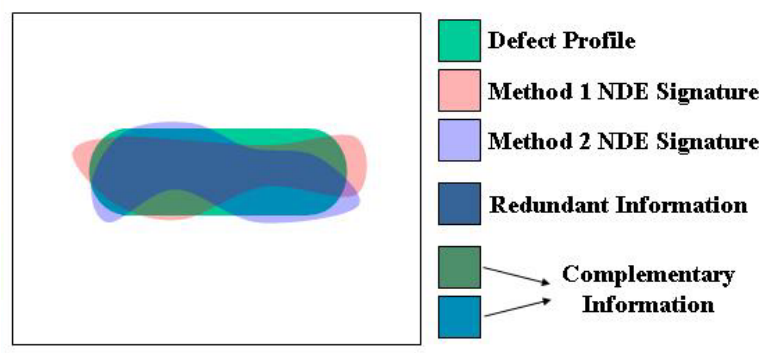

Figure 2: NDE image signatures used to define redundant and complementary information.

Typical test data results for the various pairs of NDE image combinations can be seen in Figures 3 - 5. These results are obtained using those input images that are not seen by the data fusion algorithm during the training process. The results show the inputs, outputs, and desired outputs in three rows respectively. It can be seen that the technique provides a close match to the desired complementary and redundant information.

\section{CONCLUSIONS}

The data fusion techniques presented in this paper operate by extracting redundant and complementary information present in multiples sets of observations, specifically, NDE interrogations of defects in a test specimen suite. The data fusion algorithm is sufficiently general, in that it does not specify what features in the NDE signatures are redundant or complementary - that opportunity is left to the user of the algorithm. In order to extract such information it is essential that the artificial neural network that lies at the heart of the data fusion algorithm be trained with a sufficient diversity NDE signatures that is indicative off all typical anomalies encountered in the practice of in-line inspection of gas transmission pipelines.

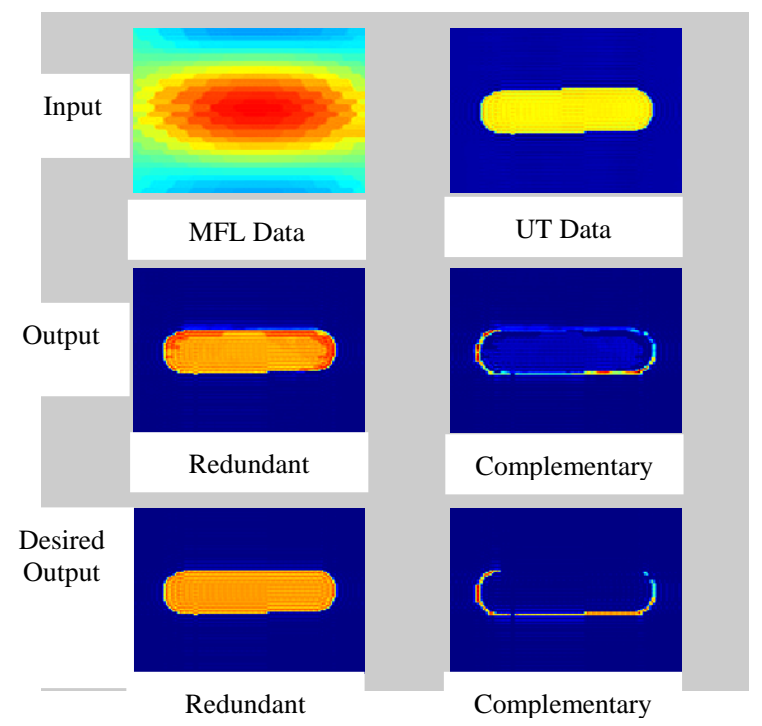

Figure 3: MFL \& UT data fusion.

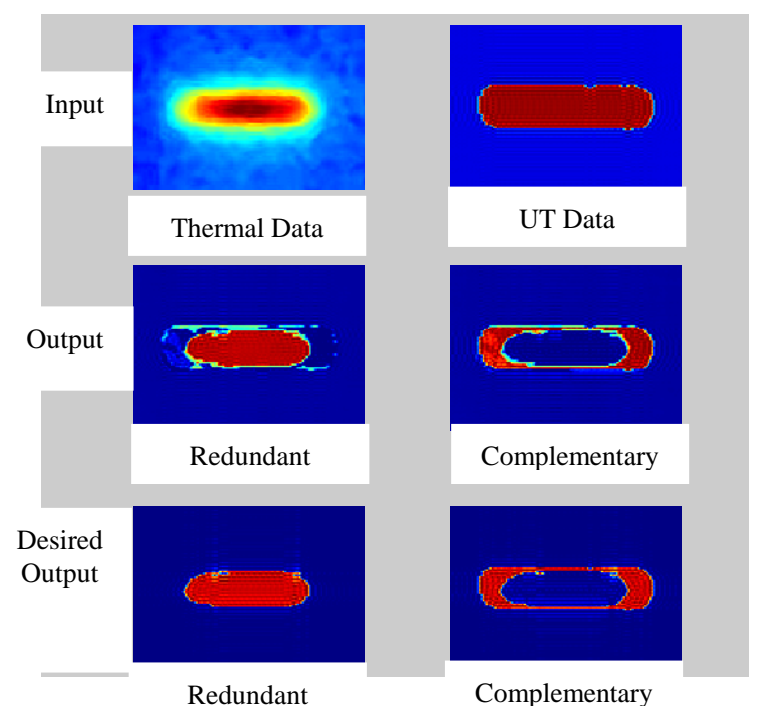

Figure 4: Thermal \& UT data fusion. 


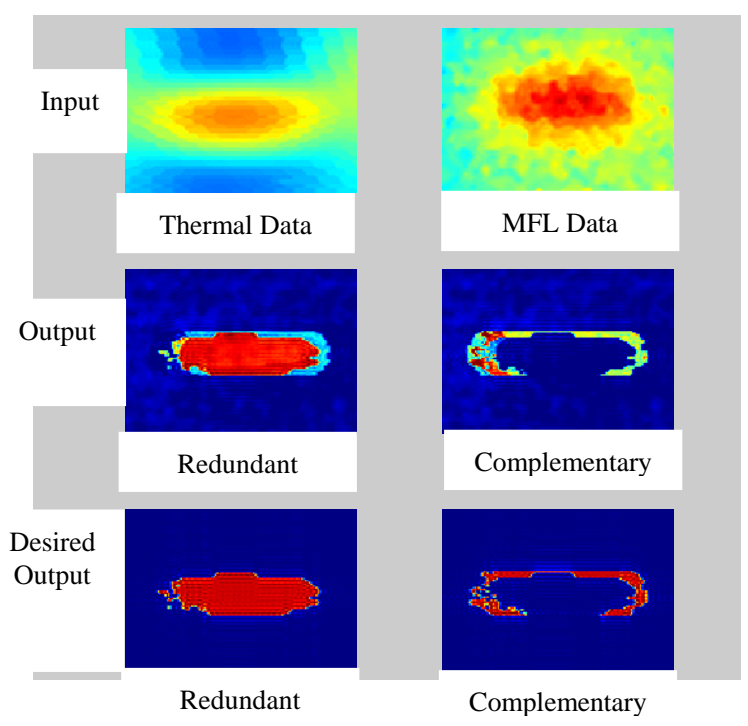

Figure 5: Thermal \& MFL data fusion.

\section{ACKNOWLEDGEMENTS}

This is work is supported by the US Department of Energy grant DE- FC26-02NT41648.

\section{REFERENCES}

[1] Don E. Bray and Roderic K. Stanley, Nondestructive Evaluation: A Tool in Design, Manufacturing, and Service, CRC Press, Boca Raton, Florida, 1997.

[2] M. Mina, J. Yim, S. S. Udpa, L. Udpa, W. Lord and K. Sun, "Two-dimensional multi-frequency eddy current data fusion," Review of Progress in Quantitative Nondestructive Evaluation, Vol. 15, Plenum Press, New York, pp. 2125-2132, 1996.

[3] X. E. Gros, J. Bousigue and K. Takahashi, "NDT data fusion at pixel level," NDT \& E International, Vol. 32, Issue 5, pp. 283-292, July 1999.

[4] D. Horn and W. R. Mayo, "NDE reliability gains from combining eddy-current and ultrasonic testing," NDT \& E International, Vol. 33, Issue 6, pp. 351362, September 2000.

[5] J. Yim, S. S. Udpa, M. Mina, L. Udpa, "Optimum filter based techniques for data fusion," Review of Progress in Quantitative Nondestructive Evaluation, Vol. 15, Plenum Press, New York, 1996, pp. 773780.

[6] Y.W. Song, NDE Data Fusion Using Morphological Approaches, PhD Dissertation, Iowa State University, Ames, Iowa, 1997.
[7] R. C. Gonzalez and R. E. Woods, Digital Image Processing, Prentice-Hall, Upper Saddle River, New Jersey, 2002.

[8] S. Mandayam, "Invariance algorithms for nondestructive evaluation," International Conference on Acoustics, Speech and Signal Processing, Salt Lake City, UT, May 2001. 